\title{
Neue HP-Therapie bei zunehmender Resistenz
}

\author{
Angesichts der wachsenden Prä- \\ valenz clarithromycinresistenter \\ Stämme von Helicobacter pylori (HP) \\ sollte eine neue Quadrupel-Behand- \\ lung als Erstlinientherapie erwogen \\ werden.
}

- Etwa $20-50 \%$ der Menschen in den Industrieländern sind Träger von Helicobacter pylori (HP), in Entwicklungsländern erreicht diese Rate bis zu $80 \%$. HP gilt als Kofaktor für eine Reihe von gastrointestinalen Erkrankungen, insbesondere dem peptischen Ulkus, dem Magenkarzinom und dem MALT-Lymphom, so dass eine Eradikation des Keims zumindest in Ländern mit hoher Inzidenz des Magenkarzinoms befürwortet wird.

In den meisten Leitlinien wird eine Behandlung bestehend aus einem Protonenpumpenhemmer und der Kombination von Amoxicillin und Clarithromycin als Standardtherapie empfohlen,

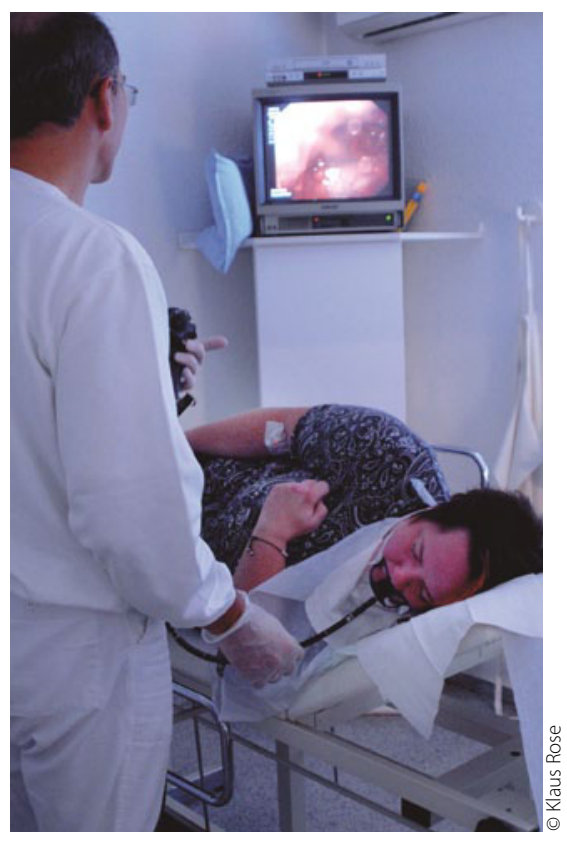

Vom Geschwür bis zum Magenkarzinom: Helicobacter gilt als Kofaktor.

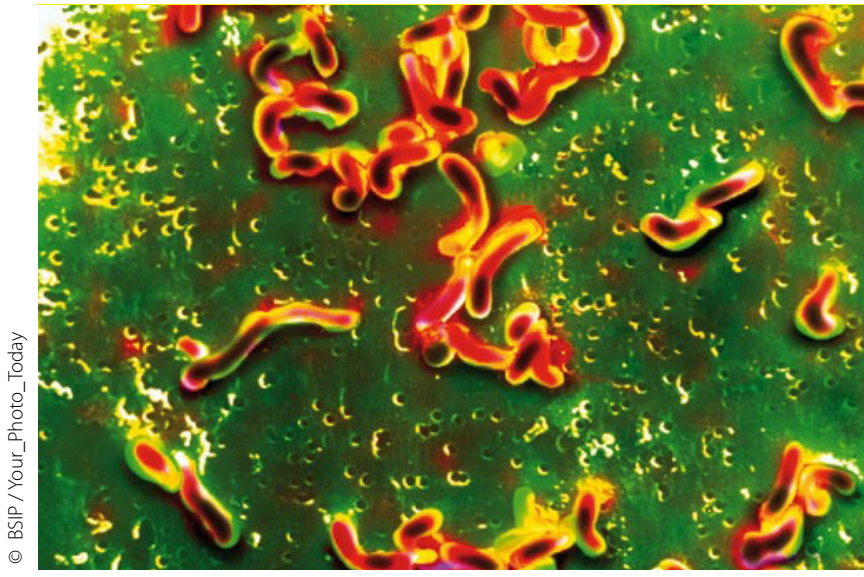

Bei bis zu $40 \%$ der HelicobacterPatienten versagt die herkömmliche Tripletherapie. sofern die Clarithromycin-Resistenz unter $20 \%$ liegt. Diese Voraussetzung ist aber immer häufiger nicht gegeben, sodass man alternative Therapien benötigt.

In einer randomisierten offenen Nichtunterlegenheitsstudie an 39 Zentren in Europa wurden Effektivität und Sicherheit einer zehntägigen Quadrupeltherapie an erwachsenen HP-Trägern - bestehend aus Omeprazol in Kombination mit Tetrazyklin, Metronidazol und Wismut-Subcitrat - gegen eine Standardtherapie aus Omeprazol, Amoxicillin und Clarithromycin über sieben Tage hinweg verglichen. Therapieziel war die Eradikation von HP, ausgewiesen durch zwei negative ${ }^{13} \mathrm{C}$-Atemtests im Abstand von mindestens 28 und 56 Tagen nach Beendigung der Therapie.

In der Analyse nach der Behandlungsabsicht gelang nach der Quadrupel-Therapie bei $80 \%$ (174 von 218) der Probanden eine Eradikation, während dies nach Standardtherapie nur bei 55\% (123 von 222) der Fall war. Unerwünschte Wirkungen bezogen sich im Wesentlichen auf Magendarmstörungen und ZNS-bedingte Beschwerden, waren aber in beiden Gruppen ähnlich verteilt.

\section{Kommentar}

Bei einem Therapieversagen von bis zu 40\% der Patienten in einigen europäischen Ländern werden alternative Behandlungsformen gegen HP dringend benötigt. Wismutpräparate wurden in der Frühzeit der HPTherapie bereits häufig verwendet, erfreuten sich wegen einiger toxischer Wirkungen dieses Schwermetalls jedoch nie großer Beliebtheit. Mit der in der Studie verwendeten Formulierung von kolloidalem Wismut-Subcitrat wurden zu keinem Zeitpunkt toxische Wismutkonzentrationen erreicht. In Ländern mit einem hohen Anteil von clarithromycin- resistenten Stämmen von HP sollte die Quadrupel-Therapie als Erstlinienbehandlung in Erwägung gezogen werden.

H. S. FÜESSL —

\section{- P. Malfertheiner et al.}

(Otto-von-Guericke Universität, 39120 Magdeburg ; peter.malfertheiner@med.ovgu.de): Helicobacter pylori eradication with a capsule containing bismuth subcitrate potassium, metronidazole, and tetracycline given with omeprazole versus clarithromycin-based triple therapy: a randomised, open-label, non-inferiority trial, phase 3. Lancet 37 (2011) 9769, 905-913. 